\title{
Conceptions et postures des enseignants du primaire à propos
} de l'intelligence

\author{
Conceptions and profiles of primary school teachers about \\ intelligence \\ Concepciones y posiciones de los maestros de primaria a \\ propósito de la inteligencia
}

\section{Élisabeth Issaieva et Marcel Crahay}

Volume 40, numéro 1, 2014

Texte reçu le : 23 septembre 2012, version finale reçue le : 14 avril 2014, accepté le : 26 juin 2014

URI : https://id.erudit.org/iderudit/1027626ar

DOI : https://doi.org/10.7202/1027626ar

\section{Aller au sommaire du numéro}

Éditeur(s)

Revue des sciences de l'éducation

ISSN

0318-479X (imprimé)

1705-0065 (numérique)

Découvrir la revue

Citer cet article

Issaieva, É. \& Crahay, M. (2014). Conceptions et postures des enseignants du primaire à propos de l'intelligence. Revue des sciences de l'éducation, 40(1), 129-156. https://doi.org/10.7202/1027626ar
Résumé de l'article

L'importance des conceptions de l'intelligence des enseignants est souvent évoquée dans les écrits de recherche mais peu explorée de manière empirique. En s'inspirant de différents courants qui abordent la question de l'intelligence et de son développement, cette recherche se propose d'investiguer les conceptions des enseignants du primaire avec l'aide d'un questionnaire et selon deux problématiques complémentaires : d'abord, en s'intéressant à la structuration de leurs conceptions et en recourant à des analyses factorielles (exploratoires et confirmatoires), et ensuite en examinant le positionnement qu'ils adoptent face aux conceptions ainsi identifiées, en réalisant des analyses typologiques. Les résultats montrent que les enseignants conçoivent l'intelligence selon cinq dimensions distinctes, mais qu'ils sont enclins à les combiner pour les intégrer dans leur posture personnelle. L'intérêt de ces résultats est discuté en fin d'article, ainsi que l'utilité du questionnaire élaboré en vue d'appréhender les conceptions de l'intelligence des enseignants. 


\section{Conceptions et postures des enseignants du primaire à propos de l'intelligence*}

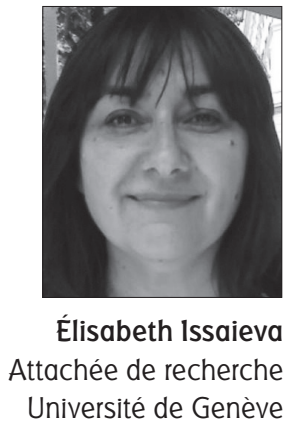

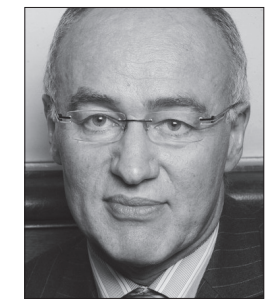

Marcel Crahay

Professeur

Université de Genève

RÉSUMÉ - L'importance des conceptions de l'intelligence des enseignants est souvent évoquée dans les écrits de recherche mais peu explorée de manière empirique. En s'inspirant de différents courants qui abordent la question de l'intelligence et de son développement, cette recherche se propose d'investiguer les conceptions des enseignants du primaire avec l'aide d'un questionnaire et selon deux problématiques complémentaires: d'abord, en s'intéressant à la structuration de leurs conceptions et en recourant à des analyses factorielles (exploratoires et confirmatoires), et ensuite en examinant le positionnement qu'ils adoptent face aux conceptions ainsi identifiées, en réalisant des analyses typologiques. Les résultats montrent que les enseignants conçoivent l'intelligence selon cinq dimensions distinctes, mais qu'ils sont enclins à les combiner pour les intégrer dans leur posture personnelle. L'intérêt de ces résultats est discuté en fin d'article, ainsi que l'utilité du questionnaire élaboré en vue d'appréhender les conceptions de l'intelligence des enseignants.

MOTS CLÉS - conceptions de l'intelligence des enseignants, échelle, validation, croyances des enseignants, intelligence.

\section{Introduction et problématique}

Désignées comme un facteur motivationnel important dans les situations d'apprentissage, les conceptions que les élèves se forgent à propos de la nature de l'intelligence, connues sous l'appellation de théories implicites, ont fait l'objet de nombreuses recherches ces dernières années (Dweck, 1986, 1999, 2010; Dweck et Leggett, 1988). Bien que les écrits sur ce sujet soient abondants, les problématiques traitées peuvent globalement s'inscrire dans deux directions. La première

\footnotetext{
* Cette recherche a été rendue possible grâce au financement du FNS (Fonds national Suisse), décision 100013_132218/1
} 
réunit les travaux qui tentent de déterminer la place occupée par les croyances relatives à l'intelligence au sein du système motivationnel des élèves, mais aussi l'influence de ces croyances sur leurs stratégies d'apprentissage, leur façon d'aborder les tâches scolaires et, finalement, leurs acquis. Les recherches menées dans cette perspective font apparaitre des résultats devenus classiques: les élèves qui croient que l'intelligence est une caractéristique stable et non contrôlable tendent à adopter des buts de performance, à douter de l'utilité de faire des efforts et, considérant les erreurs comme des signes d'incompétence, ils évitent les problèmes difficiles susceptibles de les engendrer, persévèrent moins en cas d'échec, ou encore, sous-estiment leurs capacités; quant aux élèves qui croient que l'intelligence peut évoluer, ils tendent à se centrer davantage sur la compréhension des concepts que sur les résultats à obtenir, ils n'ont pas peur d'affronter les difficultés, les surmontent grâce à leurs efforts et, in fine, obtiennent de meilleures résultats (Blackwell, Trzeniewski et Dweck, 2007; Dweck, 1999; Dweck et Molden, 2005; Elliott et Dweck, 1988; Elliot et Harackiewicz, 1996; Hong, Chiu, Dweck, Lin et Wan, 1999; Robins et Pals, 2002).

Une seconde direction pour les recherches s'est donné pour objets d'étude l'origine et le développement des conceptions de l'intelligence des élèves (Da Fonseca, Cury, Bailly et Rufo, 2004; Dweck, Chiu et Hong, 1995; Schunk, 1995). Malgré l'intérêt affiché par les chercheurs pour cette question, les investigations réalisées restent à ce jour peu nombreuses et principalement de type expérimental. Leurs résultats apportent néanmoins des éclairages quant aux facteurs qui peuvent expliquer l'élaboration de différentes conceptions chez les élèves. Ainsi, les commentaires évaluatifs des adultes (parents, enseignants), ou encore, le contenu de l'enseignement semblent affecter les significations que les élèves attribuent au concept d'intelligence: les élèves qui reçoivent des rétroactions centrées sur leurs capacités adhèrent à l'idée que l'intelligence est un attribut personnel fixe et non contrôlable, tandis que ceux qui reçoivent des rétroactions pour leur efforts, ou suivent un enseignement portant sur la malléabilité des capacités cognitives, souscrivent à l'idée que l'intelligence a un caractère dynamique et contrôlable (Blackwell et al., 2007; Butler, 2000; Kamins et Dweck, 1999). Ces résultats indiquent donc que les croyances des élèves en matière d'intelligence sont influencées par certaines pratiques d'enseignement. Ces résultats incitent également à se demander pourquoi certains enseignants portent des jugements centrés sur les capacités, alors que d'autres expriment des jugements constructifs encourageant les efforts et le travail. L'une des hypothèses qu'on peut avancer à ce sujet consiste à supposer que la différenciation dans la nature des jugements est tributaire des significations et du sens que les enseignants accordent à l'intelligence; bref, de leurs croyances en ce domaine ou encore des théories implicites qu'ils en forment. Ce qui nous conduit à faire l'hypothèse d'un lien entre les croyances des enseignants concernant l'intelligence et certaines de leurs pratiques d'enseignement, notamment leurs commentaires évaluatifs. 
Cette hypothèse générale n'est pas neuve. En effet, bien qu'elle ne soit pas explorée de manière systématique, l'importance des conceptions de l'intelligence chez les enseignants est clairement mise en avant dans les écrits des chercheurs (Crahay, 2007; Doudin et Martin, 1999). Ainsi, diverses recherches portant sur les pratiques et les croyances à l'égard du redoublement ou de la difficulté scolaire laissent penser que celles-ci sont souvent le reflet de l'adhésion des enseignants à une conception maturationniste de l'intelligence, fondée sur l'idée que les enfants sont dotés de capacités et de rythmes de développement différents (Allal et Schubauer-Leoni, 1993; Berzin et Brisset, 2008; Burdevet, 1994, Crahay, 2007; Smith 1990, Crahay, 2007). Dans le même ordre d'idée, d'autres travaux (Bressoux et Pansu, 2003; Marcoux et Crahay, 2008) ont permis de constater que dans le processus décisionnel menant au redoublement, les enseignants recourent à un jugement normatif qui s'appuie sur des heuristiques concernant des dimensions psychologiques, telles que le manque de maturité ou de capacités; ce qui laisse penser, comme le suggèrent Marcoux et Crahay (2008), que la décision en faveur du redoublement repose en grande partie sur une conception du développement de type maturationniste ou fixiste. Doudin et Martin (1999) résument bien comment deux croyances opposées de l'intelligence peuvent mener à une série d'attributions (en matière d'erreur, de causes de la difficulté scolaire, de styles d'enseignement, de rôle de l'enseignant) et de mesures pédagogiques différenciées. Il semble notamment que les enseignants qui adhèrent à une conception innéiste (capacités intellectuelles vues comme fixes et héréditaires) de l'intelligence considèrent que les erreurs et les difficultés scolaire des élèves sont inhérentes à leurs caractéristiques individuelles et, partant, que leur marge de manœuvre au niveau de l'enseignement est limitée. En revanche, les enseignants qui souscrivent à une conception constructiviste et interactionniste (supposant que l'intelligence s'élabore à travers les interactions avec l'environnement matériel et social) attribuent un rôle positif à l'erreur et s'estiment capables de combattre les difficultés scolaires, qui relèvent pour eux principalement du manque d'effort. De plus, pour ces auteurs, comme pour Crahay (2007), l'influence de ces conceptions dépasserait le cadre du fonctionnement pédagogique de l'enseignant pour atteindre celui des élèves.

Sans doute, l'opposition inné-acquis se retrouve-t-elle au cœur de la réflexion de la plupart, sinon de tous les enseignants, mais cette distinction nous paraît incomplète et insuffisante pour rendre compte des significations que les adultes peuvent accorder à un concept aussi complexe et pluridimensionnel que l'intelligence (Fournier et Lécuyer, 2006). Les résultats d'une recherche récente de Mickovska (2009) montrent le bien-fondé de cette idée. Tout en partant de la conceptualisation de Dweck (1999), selon laquelle les individus peuvent élaborer deux théories implicites opposées eu égard à l'intelligence (soit la théorie de l'entité, soit la théorie de la malléabilité), cette chercheuse met en évidence que les enseignants peuvent adopter trois postures face à l'intelligence: innéiste, incrémentaliste ou environnementaliste, mais également mixte. Pour appréhender 
si et comment les enseignants perçoivent et distinguent les différents aspects de l'intelligence, il nous semble donc nécessaire de dépasser l'approche dichotomique développée par Dweck (1999) et d'envisager une perspective qui prenne en compte la pluralité du concept d'intelligence et la complexité des facteurs pouvant contribuer à son développement. Pour ce faire, nous nous inspirerons évidemment de la conceptualisation élaborée par Dweck, ainsi que de différents courants de recherche et théories selon lesquels l'intelligence est un construit qui s'organise autour de son origine, et aussi de sa nature unie ou multidimensionnelle, de ses formes, ou encore, de ses facteurs de développement.

La recherche de Mugny et Carugati (1985), l'une des rares ayant exploré le spectre des représentations de l'intelligence chez les enseignants, montre le bien-fondé de cette remarque. En conduisant des analyses factorielles exploratoires, cette recherche pionnière fait apparaître une pluralité de conceptions. Parmi celles-ci, on trouve des conceptions de types innéiste et constructiviste, mais aussi d'autres renvoyant à d'autres aspects, formes ou fonctions de l'intelligence et de son développement, comme la pluralité des formes d'intelligence, les compétences sociales ou culturelles, les capacités d'adaptation, le type de processus cognitifs mobilisés, la vitesse d'apprentissage, etc. Cependant, il importe de rappeler que le questionnaire élaboré et utilisé dans le cadre de cette recherche se base sur de nombreuses thématiques hétérogènes. Aussi, ces chercheurs analysent de manière distincte les représentations de la notion de l'intelligence et celles de son développement. Cela a pour conséquence que certaines des conceptions mises en évidence se chevauchent et que la structure factorielle ne paraît pas totalement nette. Certains facteurs ne sont pas faciles à interpréter. Malgré ses limites, cette recherche définit, à nos yeux, un champ d'investigation qui mérite d'être exploré si l'on veut clarifier l'intervention des conceptions de l'intelligence des enseignants dans les processus d'enseignement et d'apprentissage.

\section{Cadrage conceptuel}

En tenant compte de la réflexion développée ci-dessus, nous émettons l'hypothèse que les conceptions des enseignants reflètent d'une certaine manière la pluralité des débats scientifiques à propos du concept d'intelligence. Non pas que nous postulions une influence directe des controverses entre chercheurs sur les représentations sociales, mais plutôt parce que nous supposons des interactions sous forme de va-et-vient entre les convictions de sens commun et les théories à prétention scientifique. Si l'on se réfère à des auteurs comme Dweck (1999), Gardner (1997), Grégoire (2000), Larivée (2007), Larivée et Morin (2007), Sternberg (2000), Sternberg, Lautrey et Lubbart (2003), il semble que l'on puisse distinguer quatre thématiques principales eu égard à ce concept: (1) celle de l'origine, (2) celle de la nature de l'intelligence; la question de l'origine débouchant ensuite sur (3) celle des facteurs de développement et celle de la nature de l'intelligence sur la question des (4) formes ou styles d'intelligence. 
La question de l'origine oppose principalement les innéistes aux environnementalistes, mais peut également trouver une position médiane chez ceux qui optent pour une conception interactionniste. De surcroît, dès lors que l'on laisse une place à l'influence du milieu, se pose la question de la forme de cette influence. À cet égard, on peut distinguer une conception cumulative ou behavioriste qui suppose que l'intelligence résulte de l'accumulation de connaissances ou, au contraire, adopter un point de vue constructiviste postulant que le développement intellectuel résulte d'un processus de type dialectique dans lequel l'enfant améliore ses capacités cognitives au travers de ses échanges adaptatifs avec le milieu. Dans cette perspective constructiviste, on peut faire une place plus ou moins grande aux interactions sociales, ainsi qu'à l'influence des codes sociaux et de la motivation. Appliquées aux conceptions des enseignants, ces questions nous amènent à supposer que ces derniers peuvent penser que les capacités intellectuelles sont fixées dès la naissance, liées à des dons et immuables, ou qu'elles sont évolutives (Dweck, 1999). S'ils considèrent que ces capacités sont évolutives, ils peuvent essayer d'expliquer cette évolution par différents paramètres et processus. Ainsi, en recourant au courant béhavioriste, ils peuvent considérer l'évolution comme résultant de l'accumulation de connaissances. En référence à la théorie constructiviste de Piaget (Piaget et Inhelder, 1966) et à la théorie socioconstructiviste de Doise et Mugny (1981), ils peuvent envisager que l'intelligence se développe à travers un processus d'échanges constructifs avec l'environnement matériel et social. Ils peuvent encore souscrire à la perspective historico-culturelle de Vygotsky (1978) et penser que la genèse du développement est ancrée dans le milieu culturel et qu'elle émerge dans la médiation avec l'adulte (l'enseignant), dont le rôle est prédominant. Certains enseignants peuvent aussi accorder une priorité aux facteurs motivationnels et adhérer à l'idée que le développement trouve sa source dans un climat affectif propice et confiant.

La problématique de la nature de l'intelligence oppose essentiellement les conceptions unitaristes qui consistent à supposer qu'il existe une capacité cognitive d'ordre général, correspondant notamment au facteur $\mathrm{G}$ de Spearman (Fournier et Lécuyer, 2006), et les conceptions selon lesquelles l'intelligence peut prendre des formes différentes (Gardner, 1997). Entre ces deux positions extrêmes, on peut trouver une conception de compromis qui, à l'image du modèle de Carroll (1993), considère que l'intelligence est constituée d'une capacité générale combinée à des capacités spécifiques de second ordre. Bref, lorsqu'il est question des enseignants, on peut supposer que certains conçoivent l'intelligence comme une capacité générale, tandis que d'autres pensent qu'elle se présente sous différentes formes, certaines plus développées que d'autres chez la même personne, tandis que d'autres encore se la représentent, en accord avec le modèle de Carroll (1993), comme une capacité générale composée d'autres capacités plus spécifiques mais interliées (vitesse cognitive générale, mémoire générale, vitesse de traitement générale), et estimeraient par conséquent qu'elle possède une nature multidimensionnelle. 


\section{Objectifs}

La présente recherche se centre donc sur l'étude des conceptions que les enseignants ont de l'intelligence et de son développement. Nous distanciant de la conceptualisation finalement simplificatrice de Dweck (1999), notre approche repose sur l'idée que le concept d'intelligence étant complexe, les conceptions des enseignants à son propos ne peuvent se situer sur un continuum unidimensionnel bipolaire. Pour saisir cette notion fréquemment utilisée dans les discours psychologiques et pédagogiques, les enseignants ont recours - pensons-nous - à différents courants d'idées ou théories scientifiques diffusés soit par les médias, soit lors de conférences ou au cours de cycles de formation. Quelles idées se sont-ils appropriés? Quelle articulation en font-ils? Leur discours est-il cohérent, prenant dès lors la forme d'une théorie personnelle ou, au contraire, s'agit-il d'un simple assemblage? De ces questions se dégage une première problématique qui concerne la structuration des croyances. Dans l'étude que nous avons réalisée, nous considérons le construit conceptions de l'intelligence comme un ensemble d'inférences et de croyances renvoyant aux diverses composantes du concept. Celles-ci peuvent être regroupées en grappes thématiques ou former des théories implicites, c'està-dire des idées acquises de manière inconsciente et tacite jusqu'au moment où l'individu est incité à les faire émerger ou à les expliciter (Fiorilli, Doudin, Lafortune et Albanese, 2012). Pour formuler les items du questionnaire présenté aux enseignants (voir méthode), nous nous sommes inspirés des théories brièvement rappelées dans le cadre conceptuel. Une première interrogation se pose d'emblée: les patrons de réponses des enseignants s'apparentent-ils aux regroupements théoriques qui ont inspiré la construction du questionnaire ou, au contraire, dessinent-ils une autre organisation? Si oui, laquelle?

Si l'on se réfère aux théories implicites sur l'intelligence développées par Dweck (1999), il apparaît que les enseignants adoptent des positionnements différents face à la thématique de l'origine de l'intelligence. Nous formulons l'hypothèse que cette idée peut être étendue aux autres thématiques qui touchent à ce concept et, donc, qu'il est possible d'identifier des groupes d'enseignants se distinguant par leurs croyances relatives à l'intelligence.

De façon plus précise, nous ciblons l'investigation des conceptions des enseignants selon deux problématiques complémentaires: d'abord, en nous intéressant à la structuration de leurs conceptions et ensuite en examinant le positionnement qu'ils adoptent face aux conceptions ainsi identifiées. Pour ce faire, dans l'analyse de résultats qui suivra, nous adopterons deux modalités de traitement statistique: d'une part, des analyses factorielles pour appréhender comment les enseignants distinguent et combinent les conceptions relatives à l'intelligence et, d'autre part, des analyses de classification pour voir avec quelles théories les enseignants sont en accord. 


\section{Méthode}

\subsection{Instrumentation}

Afin d'appréhender les conceptions de l'intelligence des enseignants, nous avons élaboré un questionnaire rassemblant des propositions qui renvoient aux divers aspects du concept d'intelligence comme nous les avons exposés dans le cadre conceptuel. De façon précise, les 45 items peuvent être regroupés selon qu'ils traitent de quatre thématiques principales: (1) origine de l'intelligence, (2) modalités de développement de l'intelligence, (3) nature uni ou multidimensionnelle de l'intelligence, (4) formes ou styles d'intelligence.

- Le premier domaine thématique a trait à l'origine de l'intelligence. Il s'inspire du modèle de Dweck (1999) qui véhicule l'idée que l'intelligence peut être perçue soit comme une aptitude générale héréditaire et statique, soit comme une qualité dynamique qui évolue. D’après Dweck, ces deux manières opposées de percevoir l'intelligence seraient liées à la notion d'effort. Si un individu pense que l'intelligence est un trait immuable, il peut considérer l'effort comme vain et inutile. En revanche, s'il considère que l'intelligence est une capacité qui peut s'acquérir grâce au travail, il verra l'effort comme un moyen de développement. Pour appréhender dans quelle mesure les répondants s'accordent ou non avec les aspects cités, nous avons distingué deux dimensions:

- la première dimension renvoie à deux conceptions opposées de l'intelligence: l'une innéiste et l'autre, malléable. Les 10 items servant à mesurer l'une ou l'autre des conceptions s'inspirent à la fois de l'échelle de Dweck (1999) et du questionnaire de Mugny et Carugati (1985).

- la seconde dimension renvoie à la notion de l'effort. Elle regroupe 3 items permettant de mesurer l'importance qui lui est attribuée.

- Le deuxième domaine de l'échelle vise à cerner ce que les individus pensent du développement de l'intelligence et dans quelle mesure certains facteurs le favorisent. Il comporte une série d'items qui peuvent être regroupés en quatre dimensions.

- La $1^{\text {re }}$ dimension (6 items) s'appuie sur le courant behavioriste selon lequel le développement intellectuel résulte de l'accumulation de connaissances.

- La $2^{\mathrm{e}}$ dimension (5 items) reflète la conception socioconstructiviste selon laquelle le développement intellectuel survient à travers les multiples interactions actives de l'individu avec l'environnement physique et autrui (conflit socio-cognitif).

- La $3^{\mathrm{e}}$ dimension (3 items) attribue une place prépondérante aux contextes, outils et contenus culturels dont l'influence est médiatisée par l'enseignant qui joue un rôle déterminant pour le développement de l'intelligence. On peut donc la situer dans la perspective historico-culturelle développée par Vygotsky (1978).

- Selon la $4^{\text {e }}$ dimension, le développement de l'intelligence est conçu comme le produit d'un ensemble de facteurs motivationnels d'origines sociale et personnelle (3 items). Autrement dit, elle accorde à la fois un rôle aux 
attentes de l'entourage (Rosenthalet Jacobson, 1971) et aux facteurs motivationnels personnels, tels que la confiance et l'estime de soi (Bandura, 1986; Harter, 1982).

- Le troisième domaine thématique s'inspire du modèle hiérarchique de Carroll (1993), synthèse de plusieurs modèles ayant pour point commun de considérer la nature de l'intelligence comme multidimensionnelle. En accord avec ce postulat, nous admettons que l'intelligence serait perçue comme un ensemble constitué d'une composante générale se déclinant en capacités spécifiques (mémoire et capacité de compréhension, etc.). Ce domaine thématique comporte en tout 12 items et se divise en 2 dimensions : mémoire (6 items) et vitesse cognitive (6 items), cette dernière résultant de la plus ou moins grande capacité de compréhension du sujet.

- Le quatrième domaine thématique ciblé se base sur la théorie développée par Gardner (1997). Selon cette théorie, il existerait des formes (styles ou types) multiples d'intelligence (langagière, logicomathématique, interpersonnelles) prédominantes chez certains individus et non chez d'autres (3 items).

Cette structuration est résumée dans le tableau qui suit.

Le questionnaire articulé autour de ces domaines thématiques se présente sous

Tableau 1

Composition du questionnaire

\begin{tabular}{|c|c|c|}
\hline Thématiques & Conceptions envisagées & $\begin{array}{l}\text { Nombre } \\
\text { d'items }\end{array}$ \\
\hline \multirow{3}{*}{ Origine } & Caractère immuable ou malléable de l'intelligence & 10 \\
\hline & & items \\
\hline & Place de l'effort & 3 items \\
\hline \multirow{5}{*}{$\begin{array}{l}\text { Développement de } \\
\text { l'intelligence }\end{array}$} & Conception behavioriste: accumulation de connaissances & 6 items \\
\hline & $\begin{array}{l}\text { Conception socioconstructiviste : le développement intellectuel } \\
\text { survient à travers les multiples interactions actives de l'individu }\end{array}$ & 5 items \\
\hline & avec l'environnement physique et social & \\
\hline & $\begin{array}{l}\text { Conceptions vygotskienne: importance des contextes, outils et } \\
\text { contenus culturels dont l'influence est médiatisée par l'enseignant }\end{array}$ & 3 items \\
\hline & $\begin{array}{l}\text { Le développement de l'intelligence est le produit d'un ensemble } \\
\text { de facteurs motivationnels d'origine sociale et personnelle }\end{array}$ & 3 items \\
\hline \multirow{2}{*}{$\begin{array}{l}\text { Conception } \\
\text { multidimensionnelle de } \\
\text { l'intelligence }\end{array}$} & L'intelligence repose sur la mémoire & 6 items \\
\hline & $\begin{array}{l}\text { L'intelligence est une capacité de compréhension, d'où une } \\
\text { vitesse cognitive plus ou moins grande }\end{array}$ & 6 items \\
\hline Formes de l'intelligence & $\begin{array}{l}\text { L'intelligence est multiple: conception similaire à la théorie de } \\
\text { Gardner }\end{array}$ & 3 items \\
\hline
\end{tabular}

forme d'échelle de Likert permettant aux répondants d'exprimer leur degré d'accord selon 4 modalités, allant de 1 (pas du tout d'accord) à 4 (tout à fait d'accord). 


\subsection{Participants}

En tout, 119 futurs enseignants du primaire en dernière année de formation à l'Institut universitaire de formation des maittres de Bonneville (département de la HauteSavoie, France) ont participé à l'étude préliminaire en vue de tester la compréhension du questionnaire élaboré par nos soins. L'échantillon comporte 105 femmes (89\%) et 14 hommes (11\%), dont l'âge moyen était de 27 ans et 6 mois (écart-type: 6 ans et 7 mois). Quant à l'étude principale, celle-ci a été réalisée auprès de 212 enseignants de 39 écoles primaires, situées dans trois circonscriptions du département de la Haute-Savoie en France. Ces enseignants enseignent dans différents degrés (du ${ }^{\mathrm{l}^{\mathrm{er}}}$ au

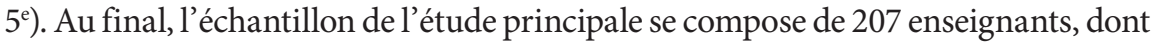
$74 \%$ de femmes et $26 \%$ d'hommes; leur âge varie de 22 à 60 ans $(M=41$ ans, $E T=9$ ans et 7 mois): 18,8\% d'entre eux ont moins de 5 ans d'expérience, $29,5 \%$ entre 5 et 15 ans d'expérience, $28,5 \%$ entre 15 et 25 ans d'expérience et $23,2 \%$ plus de 25 ans d'expérience.

\subsection{Déroulement}

L'étude préliminaire a été réalisée au sein de l'Institut universitaire de formation des maitres. Les étudiants ont rempli le questionnaire au cours d'une séance collective organisée à la fin d'un cours. Les enseignants qui participaient à l'étude principale ont été sollicités au sein de leur établissement. Un questionnaire en version papier, accompagné d'une enveloppe pour le retour, a été soumis aux enseignants volontaires. Ils nous l'ont retourné par courrier postal. Le délai de retour était d'un mois.

\subsection{Méthode d'analyse de résultats}

Afin d'affiner (des items superflus et peu clairs) le questionnaire initial, composé de 45 items, et de tester sa compréhension, nous avons réalisé une série d'analyses (descriptives et corrélationnelles) sur les données colligées auprès de l'échantillon de l'étude préliminaire. À la suite de ces analyses, le questionnaire a été réduit à 38 items et soumis à l'échantillon de l'étude principale. Ensuite, en vue de cerner les conceptions des enseignants relatives à l'intelligence et d'identifier leur structure, nous avons effectué des analyses factorielles exploratoires et confirmatoires portant sur les données de l'étude principale. Notons que cinq enseignants n’ont pas répondu à la majorité des items et ont été exclus des analyses. Par ailleurs, le pourcentage de données manquantes était inférieur à $3 \%$ et concernait divers items et modalités de réponse. De ce fait, nous n’avons pas procédé à des imputations, mais avons exclu ces items des analyses.

La fiabilité interne des facteurs apparus a été examinée dans un second temps avec l'aide du coefficient de consistance interne alpha de Cronbach ainsi que leurs corrélations. Les analyses factorielles ont été réalisées en choisissant la méthode d'extraction de maximum de vraisemblance, puisque les données présentaient des distributions qui avaient un faible écart par rapport à la normalité (Jöreskog et Long, 1993). Ainsi, les 38 items avaient tous des valeurs d'aplatissement et 
d'asymétrie très faibles (entre -1 et +1 ) et sans effet de plancher ou de plafonnement important (voir annexe 1). Supposant aussi que les conceptions peuvent être liées, nous avons opté pour une rotation oblique (Promax) lors de l'analyse factorielle. La rotation Promax permet de maximiser les corrélations entre les facteurs. Pour déterminer le nombre de facteurs à extraire, nous nous sommes basés sur deux critères: celui de Kaiser-Guttman (suggérant de conserver les facteurs ayant une valeur propre initiale supérieure de 1) et le test du Coude de Cattell (interprétation du graphique et conservation des facteurs situés avant le point d'inflexion du graphique ou rupture du coude). Les items ayant un poids factoriel inférieur à .30 et saturant sur plusieurs facteurs ont été exclus à travers des analyses successives. Aussi, pour s'assurer que les données collectées se prêtaient à l'analyse factorielle, c'est-à-dire qu'elles formaient un ensemble suffisamment cohérent et adéquat, nous avons observé les valeurs, d'une part, de l'indice de Kaiser, Meyer et Olkin et, d'autre part, du test de sphéricité de Bartlett.

Pour tester la validité du modèle structurel identifié précédemment et voir si les items (variables observées) reflètaient bien les facteurs (variables latentes) qui étaient censés les mesurer, nous avons procédé à une analyse factorielle confirmatoire (à l'aide du logiciel AMOS). Pour évaluer la pertinence du modèle testé, l'analyse se base sur des coefficients standardisés obtenus par la méthode d'estimation de maximum de vraisemblance (maximum likehood) des paramètres et les valeurs de trois indices d'ajustement, souvent conseillés ( $\chi^{2}$, CFI et RMSEA). Le $\chi^{2}$ évalue l'importance de la différence entre le modèle théorique anticipé et le modèle observé. L'ajustement de celui-ci est d'autant meilleur que le $\chi^{2}$ décroît vers 0 sans être significatif à 0,05 ; le rapport du $\chi^{2}$ et le degré de liberté ne doit pas aussi dépasser la valeur de 2. Le Comparative Fit Index (CFI) permet de comparer l'ajustement du modèle hypothétique aux modèles nuls. Il varie de 0 à 1 et montre un bon ajustement quand il atteint au moins 0,90. L'indice de l'adéquation parcimonieuse, le RootMean Square Error of Approximation (RMSEA) permet d'estimer le risque encouru que les modèles soient peu adéquats aux données. Lorsqu'il ne dépasse pas 0,05 , on peut considérer qu'il s'agit d'un bon ajustement.

Le second objectif de la présente recherche consistait à savoir comment les enseignants interrogés se positionnaient par rapport aux différentes conceptions conservées par l'analyse factorielle. Pour ce faire, nous nous sommes donc intéressés aux individus et avons conduit des analyses d'agrégation numérique à partir des scores factoriels obtenus pour chaque facteur composant la structure validée précédemment (sur les données récoltées lors de l'étude principale). En premier lieu, nous avons réalisé une analyse d'agrégation numérique hiérarchique (Hierarchical cluster analysis, méthode de Ward, le lien complet et le carré de la distance euclidienne comme mesure de distance). Ce type d'analyse offre la possibilité d'identifier des groupes (clusters) en évitant le chevauchement entre eux, surtout quand les profils qui se dégagent sont nombreux. Dans un second temps, pour confirmer quel était le regroupement en clusters le plus approprié, une analyse avec la méthode de K-moyenne a été réalisée. 


\subsection{Considérations éthiques}

Le questionnaire adressé aux enseignants était accompagné d'une lettre expliquant l'objectif de la recherche, la manière de répondre et précisant que l'étude était anonyme. Il leur a également été précisé que les données colligées resteraient confidentielles et ne serviraient qu'à des fins de recherche et qu'ils auraient un retour, s'ils le souhaitaient, dès la validation de ce travail de recherche par une publication scientifique.

\section{Résultats}

\subsection{Résultats de l'étude préliminaire}

L'étude conduite auprès de 119 futurs enseignants en vue de tester la compréhension des énoncés et d'améliorer leur formulation, nous a amenés à supprimer un item considéré comme peu clair par la majorité des participants. Il s'agit de l'item suivant: Nous naissons chacun avec un certain potentiel intellectuel, à l'image d'un réservoir délimité de capacités. Six autres items ont été supprimés à la suite à des analyses corrélationnelles: ils n'étaient pas significativement corrélés avec suffisamment d'énoncés de la dimension à laquelle ils appartenaient.

\subsection{Structuration des conceptions des enseignants}

Les analyses factorielles ont permis d'aboutir à une solution à 5 facteurs, facilement interprétables du point de vue conceptuel et réunissant 19 des 38 items du questionnaire. Les valeurs, d'une part, de l'indice de Kaiser, Meyer et Olkin $(0,68)$ et, d'autre part, du test de sphéricité de Bartlett $(\chi=1573,151, p<.001)$ montrent que les données forment un ensemble suffisamment cohérent et adéquat et que la structure factorielle extraite peut être acceptée. Cette structure à 5 dimensions permet d'expliquer en tout $57,24 \%$ de la variance totale observée (voir le tableau 2). Les 19 énoncés éliminés avaient un poids factoriel très faible sur un ou plusieurs facteurs. Il importe de rappeler que la plupart de ces énoncés non retenus étaient rattachés initialement à trois thématiques:

- celle qui traite la question de l'origine de l'intelligence (6 énoncés);

- celle qui a trait au développement de l'intelligence et concerne plus particulièrement la dimension rôle des attentes et de la motivation (3 énoncés);

- enfin, celle relative à la perception de la nature de l'intelligence (12 énoncés), dont une dimension entière n'apparaît pas dans la structure finale (lien ou non avec la mémoire).

Parmi les cinq facteurs apparus, quatre $\left(1^{\mathrm{e}}, 2^{\mathrm{e}}, 3^{\mathrm{e}}\right.$ et $\left.5^{\mathrm{e}}\right)$ présentent une bonne consistance interne (l'alpha de Cronbach variant entre 0,72 et 0,85 ). Seul le quatrième en a une plus faible, mais néanmoins acceptable: l'alpha de Cronbach est de 0,63 .

1. Le premier facteur explique $12,67 \%$ de la variance et regroupe cinq des six items relatifs à la dimension rôle des connaissances dans le développement de l'intelligence. Parmi ces items, trois nient l'importance des connaissances dans 
le développement de l'intelligence et deux leur en accordent; leurs saturations sont évidemment inverses, positives pour les uns, négatives pour les autres. Par conséquent, ce facteur peut être considéré comme un facteur bipolaire, opposant deux conceptions, l'une envisageant les connaissances comme source du développement, et l'autre ne leur attribuant pas d'importance. Nous pouvons appeler ce facteur rôle des connaissances.

2. Le deuxième facteur explique $12,77 \%$ de la variance. Il se compose de quatre items appartenant initialement à deux dimensions, relativement proches, relevant du domaine développement de l'intelligence, à savoir la dimension mettant en avant le rôle du milieu socioculturel ( 2 items), et celle attribuant un rôle aux échanges constructifs avec l'environnement ( 2 items). Dit autrement, les items qui forment ce facteur véhiculent l'idée que l'intelligence se développe grâce aux interactions actives avec l'environnement et qu'elle serait tributaire de l'influence du milieu. En somme, on voit ainsi se réunir les perspectives historicoculturelle et socioconstructiviste dont le point commun est l'interaction. C'est pourquoi on peut qualifier d'interactionniste cette manière de concevoir le développement de l'intelligence.

3. Trois items ayant trait aux formes (styles) d'intelligence se retrouvent dans le troisième facteur (expliquant $15,41 \%$ de la variance). Ils renvoient clairement à la théorie des intelligences multiples de Gardner (1997) selon laquelle les individus seraient dotés de qualités et de dons qui diffèrent en fonction des domaines (littéraire, logicomathématique, social). Par exemple, ceux qui possèdent des capacités logicomathématiques n'auraient pas de facilité dans le domaine des relations sociales et vice versa. Nous pouvons nommer ce facteur formes de l'intelligence.

4. Le quatrième facteur (expliquant $10,32 \%$ de la variance) se compose de 4 items qui renvoient à deux conceptions opposées quant à l'origine et à la malléabilité de l'intelligence: une fixiste, soutenant l'idée que l'intelligence a un caractère immuable et héréditaire, et l'autre malléable, considérant au contraire que le niveau d'aptitudes intellectuelles est le même pour tous à la naissance et que c'est le milieu qui créé les différences. On perçoit donc clairement que ces items renvoient à la fois à la théorie dichotomique de Dweck (1999) et à la théorie des inégalités naturelles, appelée encore l'idéologie $d u$ don, mise en évidence dans l'étude de Mugny et Carugati (1985). Par conséquent, on peut nommer ce facteur bipolaire: origine acquise/innée.

5. Le cinquième facteur qui recouvre $6,08 \%$ de la variance totale s'organise autour de trois items issus du troisième domaine thématique, celui que notre conceptualisation initiale rattachait au modèle de Carroll. Il serait abusif de voir dans ce cinquième facteur le reflet de la conception multidimensionnelle du psychologue américain, conception combinant l'idée d'une composante générale secondée par des capacités cognitives spécifiques. Ce facteur associe l'intelligence à la capacité de compréhension et en particulier à la vitesse cognitive. D’après les enseignants interrogés, l'intelligence se traduirait essentiellement 
par une vitesse de traitement de l'information, laquelle serait liée à la capacité de compréhension et non à la mémoire. De manière générale, ce facteur peut être nommé compréhension.

Tableau 2

Saturation des items par facteur

\begin{tabular}{|c|c|c|c|c|c|}
\hline \multirow{2}{*}{ Questions } & \multicolumn{5}{|c|}{ Facteurs } \\
\hline & 1 & 2 & 3 & 4 & 5 \\
\hline $\begin{array}{l}\text { 23. Ce n'est pas en accumulant de plus en plus de } \\
\text { connaissances qu'on développe son intelligence. }\end{array}$ & $0,84^{1}$ & 0,04 & 0,14 & 0,02 & 0,09 \\
\hline $\begin{array}{l}\text { 24. Ce n'est pas en améliorant sa culture générale qu'on } \\
\text { développe son intelligence. }\end{array}$ & 0,79 & $-0,16$ & 0,03 & $-0,04$ & 0,07 \\
\hline $\begin{array}{l}\text { 21. En augmentant sa culture générale, on développe aussi } \\
\text { son intelligence. }\end{array}$ & $-0,66$ & 0,47 & $-0,04$ & 0,14 & 0,05 \\
\hline $\begin{array}{l}\text { 22. Ce n'est pas parce qu'on lit beaucoup qu'on développe } \\
\text { son intelligence. }\end{array}$ & 0,63 & $-0,07$ & 0,08 & $-0,09$ & 0,08 \\
\hline $\begin{array}{l}\text { 20. Plus on accumule des connaissances plus on devient } \\
\text { intelligent. }\end{array}$ & $-0,58$ & 0,31 & $-0,04$ & 0,17 & 0,17 \\
\hline $\begin{array}{l}\text { 5. En discutant ses idées avec autrui, on développe son } \\
\text { intelligence. }\end{array}$ & $-0,21$ & 0,73 & 0,03 & 0,08 & 0,06 \\
\hline $\begin{array}{l}\text { 7. Interagir avec des gens compétents aide à développer } \\
\text { son intelligence. }\end{array}$ & $-0,20$ & 0,73 & 0,08 & 0,16 & 0,14 \\
\hline 42. L'intelligence se construit en interaction avec le milieu. & $-0,05$ & 0,69 & 0,11 & 0,26 & 0,17 \\
\hline $\begin{array}{l}\text { 41. L'intelligence se développe principalement grâce aux } \\
\text { stimulations du milieu. }\end{array}$ & 0,10 & 0,56 & 0,13 & 0,32 & 0,17 \\
\hline $\begin{array}{l}\text { 26. Les élèves doués pour les relations sociales sont } \\
\text { souvent moins doués en mathématiques et en sciences. }\end{array}$ & 0,09 & 0,18 & 0,97 & $-0,08$ & 0,15 \\
\hline $\begin{array}{l}\text { 25. Les élèves doués en mathématiques et en sciences } \\
\text { sont souvent moins doués pour les relations sociales. }\end{array}$ & 0,09 & 0,13 & 0,91 & $-0,11$ & 0,10 \\
\hline $\begin{array}{l}\text { 27. Les élèves qui ont une intelligence plutôt littéraire } \\
\text { éprouvent des difficultés dans les domaines scientifiques. }\end{array}$ & 0,07 & 0,00 & 0,59 & $-0,07$ & 0,17 \\
\hline $\begin{array}{l}\text { 11. À la naissance, hormis les cas de handicap sévère, tous } \\
\text { les enfants ont le même potentiel intellectuel. }\end{array}$ & $-0,06$ & 0,26 & $-0,05$ & 0,89 & $-0,25$ \\
\hline $\begin{array}{l}\text { 12. Dès la naissance, certains sont plus intelligents que } \\
\text { d'autres. }\end{array}$ & 0,04 & $-0,22$ & 0,13 & $-0,76$ & 0,27 \\
\hline $\begin{array}{l}\text { 10. Nous naissons tous approximativement avec les mêmes } \\
\text { capacités intellectuelles. C'est le milieu qui fait la différence. }\end{array}$ & $-0,12$ & 0,10 & $-0,06$ & 0,64 & $-0,15$ \\
\hline 9. L'intelligence est fixée à la naissance. & 0,10 & $-0,33$ & 0,05 & $-0,43$ & 0,18 \\
\hline $\begin{array}{l}\text { 38. Un élève intelligent est un élève qui comprend } \\
\text { rapidement. }\end{array}$ & 0,04 & 0,08 & 0,12 & $-0,26$ & 0,93 \\
\hline $\begin{array}{l}\text { 37. Les enfants intelligents n'ont pas besoin de recevoir de } \\
\text { longues explications avant de comprendre. }\end{array}$ & $-0,01$ & 0,17 & 0,10 & $-0,19$ & 0,76 \\
\hline $\begin{array}{l}\text { 39. Quand on est moins intelligent, il faut plus de temps } \\
\text { pour comprendre. }\end{array}$ & 0,10 & 0,20 & 0,29 & $-0,33$ & 0,63 \\
\hline \% de variance expliquée (pour l'ensemble de la structure & & & & & $08 \%$ \\
\hline
\end{tabular}

1. Les coefficients de saturation indiqués en gras sont les plus significatifs 
On peut également observer que certains items présentent des poids factoriels importants sur deux facteurs. Par exemple, un des items renvoyant au caractère immuable de l'intelligence se joint de manière assez logique (c'est-à-dire avec une saturation négative) aux items du facteur 2 renvoyant à l'idée opposée, c'est-à-dire que l'intelligence change grâce aux interactions avec le milieu. Deux items (saturés négativement) du facteur 1 se lient aux items du facteur 2. Ce résultat est instructif dans le sens qu'il est révélateur de la signification que les enseignants semblent attribuer à l'acquisition de la culture ou encore à l'accumulation des connaissances. Pour eux, leur rôle est important et il se joue probablement par l'entremise des échanges avec l'environnement. Dit autrement, accorder de l'importance aux connaissances ne veut pas dire exclure ou nier la part des interactions. On voit donc ici un rapprochement entre une conception de type behavioriste et une autre, constructiviste. Il convient toutefois de souligner que le poids factoriel des items communs des facteurs est toujours plus important sur l'un des facteurs d'appartenance; de ce fait, il convient d'être prudent dans l'interprétation de ces résultats.

L'analyse a également révélé que certains facteurs sont associés entre eux, sans que les corrélations aient une ampleur importante (les valeurs variant entre -0,30 et 0,31 ). Cela nous incite à penser que les enseignants élaborent des conceptions assez distinctes et claires (tableau 3), mais qu'ils ont tendance à faire des liens entre les différentes théories. Les corrélations les plus élevées sont, d'une part, entre les conceptions interaction et origine $(0,31)$ et, d'autre part, entre les conceptions compréhension et origine $(-0,31)$. Donc, plus les enseignants pensent que l'intelligence se développe grâce aux interactions, plus ils estiment que celle-ci constitue une capacité évolutive; plus ils pensent que l'intelligence est innée et plus ils l'associent à la capacité de compréhension.

\section{Tableau 3}

Corrélations entre les facteurs

\begin{tabular}{lcccc}
\hline Corrélations entre les facteurs & $\begin{array}{c}F 1 \\
\text { Rôle } \\
\text { connaissance }\end{array}$ & $\begin{array}{c}\text { F2 } \\
\text { Rôle } \\
\text { interaction }\end{array}$ & $\begin{array}{c}\text { F3 } \\
\text { Formes }\end{array}$ & $\begin{array}{c}\text { F4 } \\
\text { Origine }\end{array}$ \\
\hline F1 Rôle connaissance & & & & \\
F2 Rôle interaction & $-0,22^{* *}$ & & & \\
F3 Formes & 0,12 & $0,16^{*}$ & & \\
F4 Origine & $-0,09$ & $0,31^{* *}$ & $-0,12$ & $-0,30^{* *}$ \\
F5 Compréhension & 0,05 & $0,19^{*}$ & $0,18^{*}$ & \\
\hline
\end{tabular}

** La corrélation est significative au seuil de 0,01

* La corrélation est significative au seuil de 0,05 


\subsection{Validité du modèle structurel}

L'analyse factorielle confirmatoire a été réalisée sur la même base de données que les analyses factorielles exploratoires, c'est-à-dire sur les données colligées auprès de l'échantillon composé de 207 enseignants du primaire. Ainsi, nous avons testé un modèle à 5 facteurs latents et 19 items ou variables observées. Les items saturés sur deux facteurs à la fois ont été considérés comme le reflet seulement du facteur sur lequel leur poids factoriel est le plus important.

Au regard des différents indices d'ajustement et des coefficients de régression, les résultats de l'analyse confirmatoire montrent que le modèle testé s'ajuste de manière satisfaisante aux données (voir annexe 2); les valeurs des indices calculés sont les suivantes: $\chi=257,8$; RMSEA $=0,06$ et $\mathrm{CFI}=0,92$. Ces valeurs nous permettent de conclure que le modèle théorique posé a priori reproduit correctement les données empiriques. Il est important de préciser que ces valeurs sont obtenues lorsque cinq covariances d'erreurs de mesure ont été prises en considération, mais entre des items ayant un contenu fortement similaire et appartenant aux mêmes facteurs. L'analyse confirme également les corrélations entre certains facteurs, mais celles-ci restent, pour la plupart d'entre elles, faibles (variant entre -0,28 et $0,30)$. Les corrélations les plus fortes sont toujours entre les facteurs: origine et interaction $(0,30)$; compréhension et origine $(-0,28)$.

\subsection{Détermination des profils d'enseignants}

Lanalyse d'agrégation numérique a permis de retenir la répartition en trois profils pour classer les 172 enseignants. Ce regroupement s'est avéré optimal du point de vue de la cohésion et de la distinction des groupes en fonction de cinq facteurs (voir tableau 4, annexes 3 et 4).

Les trois groupes ont plus ou moins le même nombre d'effectifs. Les 55 enseignants ( $32 \%$ de l'échantillon) regroupés dans le groupe A rapportent le niveau le plus élevé sur le facteur rôle des connaissances et lui accordent donc de l'importance dans le développement de l'intelligence. Par ailleurs, ces enseignants semblent ne pas prendre en considération les autres facteurs. Pour eux, le développement de l'intelligence est donc essentiellement vu par le prisme des connaissances et, plus précisément, de leur accumulation.

Le groupe B comprend 56 enseignants (32,6\% de l'échantillon). Ceux-ci se caractérisent par un positionnement très favorable à l'égard du facteur intelligences multiples, puisque le score moyen pour ce facteur est le plus élevé. Ce score est également le plus important en comparaison avec les scores des autres groupes. Les enseignants du groupe B semblent aussi accorder de l'importance au facteur capacité de compréhension. Dans une moindre mesure, ils prennent en considération l'influence du milieu ainsi que les interactions, et ne semblent pas beaucoup tenir compte ni des connaissances ni de l'origine. Dit autrement, pour ces enseignants, l'intelligence se présente surtout sous des formes différentes et renvoie à la capacité de compréhension. 
Les 61 enseignants réunis dans le groupe C (35,5\% de l'échantillon) affichent le score le plus élevé au niveau du facteur «origine acquise versus innée». Cela atteste de leur forte adhésion à l'idée que l'intelligence est une caractéristique qui peut se développer. Le rôle des interactions et celui du milieu est également mis en avant. À l'égard des autres facteurs, les enseignants de ce groupe ne semblent pas manifester un positionnement favorable.

Afin d'examiner sur quels facteurs se situent exactement les différences significatives entre les trois groupes, nous avons réalisé une analyse de variance multivariée, suivie d'une structuration des moyennes (Bonferroni). Les résultats pour le test sont significatifs : $\lambda$ de Wilks $=0,160, \mathrm{~F}(10)=49,45,, p<.05)$. Les différences entre les groupes touchent tous les facteurs, mais l'effet est différent selon le facteur et les groupes considérés (voir le tableau 4).

Tableau 4

Moyennes et écart types (entre parenthèses) des scores factoriels

\begin{tabular}{lccccc}
\hline & Groupe A $(n=55)$ & Groupe B $(n=56)$ & Groupe C $(n=61)$ & $F$ & $\eta 2$ \\
\hline F1 Rôle connaissance & $0,45(0,82) \mathrm{a}$ & $-0,04(0,96) \mathrm{b}$ & $-0,37(0,83) \mathrm{b}$ & 12,53 & 0,12 \\
F2 Rôle interaction & $-0,80(0,79) \mathrm{a}$ & $0,35(0,66) \mathrm{b}$ & $0,40(0,72) \mathrm{b}$ & 49,08 & 0,36 \\
F3 Formes & $-0,48(0,69) \mathrm{a}$ & $1,00(0,70) \mathrm{b}$ & $-0,48(0,68) \mathrm{a}$ & 85,26 & 0,50 \\
F4 Origine & $-0,47(0,73) \mathrm{a}$ & $-0,37(0,81) \mathrm{a}$ & $0,77(0,66) \mathrm{b}$ & 51,46 & 0,37 \\
F5 Compréhension & $-0,05(0,84) \mathrm{a}$ & $0,61(0,81) \mathrm{b}$ & $-0,51(0,84) \mathrm{c}$ & 26,14 & 0,23 \\
\hline
\end{tabular}

* Pour chaque facteur, les moyennes avec des indices différents indiquent une différence significative au seuil de 0,05 (Bonferroni, post-hoc)

\section{Discussion des résultats}

L'interrogation principale de la présente recherche était de savoir comment les enseignants du primaire conçoivent l'intelligence et son développement. Pour ce faire, nous avons développé une échelle de mesure qui trouve ses sources dans des théories actuelles de l'intelligence. Nous avons opté pour deux modalités d'analyse: (1) l'analyse factorielle afin d'explorer la structuration des conceptions et (2) l'analyse typologique afin d'analyser le positionnement des enseignants face aux différentes conceptions de l'intelligence mises en évidence.

Les analyses factorielles ont montré que, pour les enseignants interrogés, l'intelligence ne constitue pas une entité générale, mais apparaît plutôt comme un construit pluriel qui peut être perçu sous des angles différents qui dépassent l'opposition inné-acquis privilégiée dans les écrits scientifiques. Dans ce sens, nos résultats apportent de nouveaux éclairages. De plus, les conceptions mises en évidence reflètent de manière cohérente les quatre domaines thématiques, ce qui nous permet de soutenir l'idée de la pluralité et de la cohérence des théories implicites sur l'intelligence chez les enseignants. Ainsi, deux conceptions ren- 
voient au domaine modalités de développement (facteurs 1 et 2) et trois autres recouvrent l'origine (facteur 4), la nature (facteur 5) et les formes (facteur 3). En conséquence, on peut affirmer que la conceptualisation de Dweck $(1999,2010)$ selon laquelle les capacités cognitives seraient perçues par les individus du point de vue de leur caractère immuable, ou inversement malléable, est simplificatrice. Certes, nous retrouvons cette dimension dans le facteur 4, mais elle n'est qu'une facette des conceptions des enseignants concernant l'intelligence.

Bien qu'elle reflète la pluralité du concept de l'intelligence et qu'elle s'organise autour de quatre thématiques, la structuration des conceptions dégagée n'englobe pas tous les items et dimensions théoriques envisagés. En ce qui concerne la thématique nature de l'intelligence, il est intéressant de constater que, parmi les deux dimensions ciblées, une seule est représentée; il s'agit de la capacité de compréhension de l'élève en lien avec la vitesse d'apprentissage. Aucun des items relevant de la dimension mémoire ne se retrouve dans la structure factorielle. Par conséquent, nous ne pouvons pas vraiment défendre l'idée que certains enseignants conçoivent l'intelligence comme un ensemble de capacités spécifiques couplées à une capacité générale, comme l'a soutenu Carroll (1993); plus exactement, ils ne l'associent pas à la capacité de mémoire. Par ailleurs, le fait d'associer la notion d'intelligence à la capacité de compréhension et à la vitesse cognitive rappelle les résultats des recherches sur la représentation de la difficulté scolaire (Desombre, Delelis, Antoine, Lachal, Gaillet et Urban, 2010; Do, 2007). Ces derniers montrent clairement que, pour expliquer les difficultés scolaires, les enseignants mettent de l'avant les capacités cognitives des élèves, en particulier la compréhension et la rapidité cognitive. En d'autres termes, tous ces résultats font la démonstration de l'importance que prend la notion de capacité de compréhension dans les raisonnements explicatifs des enseignants et probablement dans leurs pratiques pédagogiques, ainsi que dans leur manière de traiter les difficultés scolaires.

Une autre dimension, relevant cette fois-ci de la thématique modalité de développement, est absente de la structure factorielle: c'est celle qui envisage le développement des capacités cognitives comme la résultante des attentes manifestées à l'égard de l'élève et de facteurs motivationnels. Cela suggère que les enseignants français dissocient la notion d'intelligence de celle de motivation. Il semble donc que, pour eux, ce sont deux concepts qui n'ont pas de lien automatique.

En ce qui concerne le développement de l'intelligence, deux modalités émergent: l'une correspond à l'idée que l'intelligence est ou n'est pas en lien avec l'accumulation des connaissances, le facteur étant bipolaire; l'autre lie le développement à la quantité et à la qualité des interactions constructives avec le milieu physique et culturel. En d'autres termes, deux conceptions se distinguent à propos du développement de l'intelligence: l'une que l'on qualifiera de cumulative et l'autre d'interactionniste; ces deux conceptions distinctes ne s'opposent pas comme l'a montré l'analyse corrélationnelle. Il est intéressant de noter que la 
seconde de ces conceptions intègre deux conceptions que nous avions distinguées. En effet, le facteur 2 combine des items exprimant la conception socioconstructiviste inspirée de Piaget et d'autres reflétant la théorie de Vygotsky et soulignant la dimension historicoculturelle de l'intelligence. Bref, il semble bien que, pour les enseignants dont les scores factorisés sont élevés eu égard à cette échelle, l'interaction avec l'environnement et l'influence du milieu socioculturel relèvent de la même perspective qu'on peut qualifier d'interactionniste.

Par ailleurs, nos résultats confortent dans une certaine mesure le postulat développé par Dweck (1999), selon lequel la malléabilité et la stabilité seraient perçues par les enseignants comme des caractères dichotomiques et exclusifs. L'analyse factorielle dégage un facteur bipolaire indiquant que les enseignants interrogés les perçoivent soit comme un trait inné et statique, soit comme une qualité qui s'acquiert et caractérisé par son dynamisme. En allant dans ce sens, nos résultats s'opposent à la thèse défendue par un certain nombre de chercheurs (Anderson, 1995; Vezeau, Bouffard et Dubois, 2004; Schunk, 1995) selon laquelle les deux conceptions (statique/dynamique) seraient prises en considération par la même personne, ou encore, ne seraient pas complètement opposées. Ce résultat ne se retrouve pas dans notre recherche. La manière dont les deux conceptions faisant appel à ces aspects se positionnent dans le construit qui a été validé, en s'opposant sur le même facteur, montre que les enseignants interrogés adhèrent soit à l'une, soit à l'autre.

Les analyses factorielles ont permis de dégager une structure de conceptions selon lesquelles les enseignants pensent l'intelligence. Cela ne signifie évidemment pas que tous les enseignants interrogés ont la même représentation de ce concept multidimensionnel. D'une part, ceux-ci se positionnent de façon plus ou moins favorable ou défavorable par rapport aux cinq dimensions dégagées et, d'autre part, ils les combinent selon des logiques que l'analyse typologique (clustering) a permis d'investiguer. Dit autrement, si les analyses factorielles ont dévoilé que les enseignants pensent l'intelligence selon cinq dimensions distinctes, les résultats de l'analyse d'agrégation numérique montrent qu'ils sont enclins à les combiner de manières diverses pour les intégrer dans une sorte de théorie-posture personnelle. Il est à noter que, selon cette analyse typologique, seuls les enseignants du groupe $\mathrm{C}$ se montrent réellement mobilisés par la question de l'origine de l'intelligence. Ces enseignants, un tiers de notre échantillon, expriment une forte adhésion à l'idée que l'intelligence est une caractéristique qui peut se développer et que son développement est tributaire de la qualité et de la quantité des interactions avec le milieu physique, social et culturel. Les enseignants du groupe A, qui se caractérisent par la croyance selon laquelle le développement de l'intelligence est lié à l'accumulation de connaissances, ne manifestent pas de position marquée par rapport à la question de l'origine de l'intelligence. En définitive, le rôle des interactions et du milieu est clairement mis de l'avant dans un seul des groupes mis en évidence: le groupe C. Dans celui-ci, deux des conceptions 
mises en évidence au préalable et allant dans le même sens se rejoignent. Il s'agit de la conception origine et de la conception interaction. De manière logique et cohérente, plus les enseignants de ce groupe attribuent de l'importance à la part acquise de l'intelligence, plus ils se positionnent favorablement face aux interactions avec le milieu.

Il est par ailleurs tentant d'opposer les enseignants des groupes $\mathrm{A}$ et $\mathrm{C}$ dans la mesure où ceux du groupe $\mathrm{C}$ paraissent défendre un point de vue de type socioconstructiviste, alors que ceux du groupe A paraissent défendre une conception cumulative de l'intelligence. Enfin, les enseignants regroupés dans le groupe B se caractérisent avant tout par leur adhésion à la théorie des intelligences multiples de Gardner, à laquelle ils associent l'idée que l'intelligence est liée à la capacité de compréhension. Ces enseignants semblent aussi moins concernés par la question des facteurs contribuant au développement de l'intelligence, probablement parce qu' ils envisagent l'intelligence seulement sous le prisme des capacités cognitives, en accordant clairement une place prédominante à la prédisposition et à la variabilité interindividuelle. C'est en tout cas ce que laissent penser nos résultats concernant les enseignants du groupe $\mathrm{C}$ pour qui l'intelligence renvoie à des dispositions personnelles et peu modifiables. D'une certaine manière, ici on retrouve l'idée de Dweck (1999) que pour certains individus, l'intelligence est une aptitude fixe. Ces résultats sont également très proches de ceux des recherches qui ont mis en évidence que certains enseignants ont bel et bien des conceptions fixistes du développement (Crahay, 2007).

\section{Conclusion}

Malgré la tentative fructueuse de cette recherche pour mieux saisir la variété des conceptions que les enseignants élaborent de l'intelligence, celle-ci présente des limites qu'il importe de mentionner. Du point de vue méthodologique, nous avons proposé et validé la structure d'une échelle multidimensionnelle qui présente des qualités psychométriques prometteuses et qui peut servir à mesurer les conceptions de l'intelligence des enseignants dans le cadre des recherches ultérieures. Toutefois, nos analyses factorielles confirmatoires ont été appliquées sur le même échantillon que celui de l'étude exploratoire. Il faudrait tester la validité structurelle de l'échelle sur d'autres échantillons et dans d'autres contextes. Enfin, l'analyse typologique a permis de cerner globalement le positionnement des enseignants par rapport aux diverses conceptions de l'intelligence mises en évidence. Sur ce sujet, l'apport de la présente recherche demeure modeste. Pour mieux comprendre les postures des enseignants, il serait nécessaire de conduire des recherches qualitatives qui permettent de saisir la complexité de leur raisonnement. Plus précisément, il serait nécessaire - pensons-nous - de positionner les enseignants dans des groupes différents à partir de l'outil développé dans la présente recherche et ensuite d'interroger des enseignants appartenant à ces différents groupes afin d'investiguer comment précisément ils pensent les différentes facettes de l'intelligence et de son 
développement. Il serait également utile de pouvoir caractériser ces enseignants afin de savoir si certains paramètres tels que le genre et l'expérience professionnelle mènent à différentes postures face au concept d'intelligence. En tout cas, si on se base sur les travaux de Fiorilli et al. (2012, p. 62) montrant que les enseignants expérimentés glissent vers une vision plus défaitiste, nous pouvons avancer l'hypothèse qu'avec le temps et l'expérience, les conceptions que les enseignants ont de l'intelligence se modifient.

En mettant en évidence la pluralité des conceptions des enseignants à propos de l'intelligence, ainsi que de leur combinaison, cette recherche suggère en définitive un élargissement des dimensions à prendre en compte dès lors que l'on s'intéresse à l'influence des conceptions des enseignants sur leurs pratiques et, partant, sur l'apprentissage des élèves. Jusqu'ici, la question des relations entre les conceptions de l'intelligence et les pratiques d'enseignement a été abordée en considérant uniquement la dichotomie innéiste en opposition à constructiviste. Ainsi, dans une séquence d'apprentissage de l'orthographe, Fiorilli et al. (2012) ont montré que les enseignants qui avaient une conception innéiste ne fournissaient pas d'explications claires aux élèves en cas d'erreur, alors que ceux qui adhèraient à une conception constructiviste indiquaient aux élèves les stratégies d'apprentissage à améliorer ou à changer. À la lecture des résultats de notre analyse typologique, ce résultat semble valoir pour ceux du troisième groupe. Toujours à la lecture des résultats de notre étude, on est en droit de s'interroger sur la conception de la nature de l'intelligence (cumulative ou autre) qu'ont les enseignants nommés constructivistes par Fiorilli et al. (2012). De même, on est en droit de se demander comment les enseignants qui adhérent à une conception multiple de l'intelligence, conception inspirée de Gardner (1997), réagissent aux erreurs des élèves. Plus largement, le fait d'avoir pu observer le caractère pluriel des conceptions de l'intelligence des enseignants nous amène à nous interroger plus largement sur le lien entre ces conceptions et la façon dont ils réagissent aux erreurs. Nous sommes tentés de mettre en doute l'idée que seule la dimension origine de l'intelligence intervienne à ce propos. Nous nous interrogeons sur le rôle (propre et en combinaison avec la dimension origine) des autres dimensions mises en évidence dans le cadre de cette modeste recherche.

\section{ENGLISH TITLE - Conceptions and profiles of primary school teachers about intelligence}

SUMMARY - The significance of teachers' conceptions of intelligence has often been raised in literature but rarely explored empirically. By focussing on different streams of research covering the notion of intelligence and its development, this research seeks to examine the conceptions of intelligence among primary school teachers in France using a questionnaire and focussing on two complementary issues: first, we analyse the structure of teachers' conceptions using factor analysis (exploratory and confirmatory), and secondly, we use typological analyses to examine how teachers position themselves as regards the conceptions identified. Results show that teachers conceptualise intelligence on the basis of five distinct dimensions which they combine in order to integrate them into their personal position. The significance of these findings as well 
as the utility of the questionnaire developed to understand the conceptions of intelligence among teachers are discussed at the end of this contribution.

KEY WORDS - Teachers' conceptions of intelligence, scale, validation, teachers' beliefs, intelligence.

\section{TITULO - Concepciones y posiciones de los maestros de primaria a propósito de la inteligencia}

RESUMEN - La importancia de las concepciones sobre la inteligencia en los profesores y maestros aparece con frecuencia en la literatura, pero ha sido poco explorada de forma empírica. Inspirándose de diversas corrientes de investigación que abordan la cuestión de la inteligencia y de su desarrollo, esta investigación se propone estudiar las concepciones de los maestros de primaria con la ayuda de un cuestionario y según dos problemáticas complementarias: en primer lugar, interesándose a la estructura de sus concepciones y recurriendo a análisis factoriales (exploratorios y confirmatorios); posteriormente, examinando la posición que estos maestros adoptan frente a las concepciones así definidas, realizando análisis tipológicos. Los resultados muestran que los maestros conciben la inteligencia según cinco dimensiones diferentes, pero que tienen una inclinación a combinarlas para integrarlas en su posición personal. El interés de estos resultados se discute al final del artículo, así como la utilidad del cuestionario desarrollado en vista de aprehender las concepciones de la inteligencia de los maestros.

PALABRAS CLAVE • Concepciones de la inteligencia de los maestros, escala, validación, creencias de los maestros, inteligencia.

\section{Références}

Allal, L. et Schubauer-Leoni, M. L. (1993). Progression scolaire des élèves: le redoublement dans le contexte genevois. Théorie et pratique, 11/12, 41-50.

Anderson, C. A. (1995). Implicit theories in broad perspective. Psychological inquiry. An international journal for the advancement of psychological theory, 6(4), 286-289.

Bandura, A. (1986). Social foundations of thought and action: a social cognitive theory. Englewood Cliffs, New Jersey: Prentice-Hall.

Blackwell, L., Trzesniewski, K. and Dweck, C. S. (2007). Implicit theories of intelligence predict achievement across an adolescent transition: a longitudinal study and an intervention. Child development, 78(1), 246-263.

Berzin, C. et Brisset, C. (2008). Les représentations des enseignants spécialisés et non spécialisés. Carrefours de l'éducation, 25, 91-101.

Bressoux, P. et Pansu, P. (2003). Quand les enseignants jugent leurs élèves. Paris, France: Presses universitaires de France.

Burdevet, E. (1994). Comment les enseignants prennent-ils une décision de redoublement? Mémoire de Licence, Genève, Suisse: Université de Genève.

Butler, R. (2000). Making judgments about ability: the role of implicit theories of ability in moderating inferences from temporal and social comparison information. Journal of personality and social psychology, 78(5), 965-978.

Carroll, J. B. (1993). Human cognitive abilities: a survey of factor-analytic studies. Cambridge, United Kingdom: Cambridge University Press. 
Crahay, M. (2007). Peut-on lutter contre l'échec scolaire? (3édition). Bruxelles, Belgique: De Boeck Université.

Da Fonseca, D., Cury, F., Bailly, D. et Rufo, M. (2004). Rôle des théories implicites de l'intelligence chez les élèves en situation d'apprentissage. L'Encéphale, 30(5), 456-463.

Desombre, C., Delelis, G., Antoine, L., Lachal, M., Gaillet, F. et Urban, E. (2010). Comment des parents d'élèves et des enseignants spécialisés voient la réussite et la difficulté scolaires. Revue française de pédagogie, 173, 5-18.

Do, C. (2007). Les représentations de la grande difficulté scolaire par les enseignants. Les Dossiers, 182. Paris, France: ministère de l'Éducation nationale, de l'Enseignement supérieur et de la Recherche.

Doise, W. et Mugny, G. (1981). Le développement social de l'intelligence. Paris, France: InterÉditions.

Doudin, P.-A. et Martin D. (1999). Conception du développement de l'intelligence et formation des enseignants. Revue française de pédagogie, 126, 121-132.

Dweck, C. S. (1986). Motivational processes affecting learning. American psychologist, 41(10), 1040-1048.

Dweck, C. S. (1999). Self-theories: their role in motivation, personality and development. Philadelphia, Pennsylvania: Taylor and Francis/Psychology Press.

Dweck, C. S. (2010). Changer d'état d'esprit. Une nouvelle psychologie de la réussite. Wavre, Belgique: Mardaga.

Dweck, C. S., Chiu, C. and Hong, Y. (1995). Implicit theories and their role in judgments and reactions: a world from two perspectives. Psychological inquiry, 6, 267-285.

Dweck, C. S. and Leggett, E. L. (1988). A social-cognitive approach to motivation and personality. Psychological review, 95(2), 256-273.

Dweck, C. S. and Molden, D. C. (2005). Self-theories: their impact on competence and acquisition. In A. J. Elliot and C. S. Dweck (eds): The handbook of competence and motivation (p. 122-140). New York, New York: Guilford Press.

Elliott, E. S. and Dweck, C. S. (1988). Goals: an approach to motivation and achievement. Journal of personality and social psychology, 54(1), 5-12.

Elliot, A. J. and Harackiewicz, J. M. (1996). Approach and avoidance achievement goals and intrinsic motivation: a mediational analysis. Journal of personality and social psychology, 70, 461-475.

Fiorilli, C., Doudin, P.-A., Lafortune, L. et Albanese, O. (2012). Conceptions de l'intelligence et pratiques éducatives. Quelle est l'influence du constructivisme? Québec, Québec: Presses de l'Université du Québec.

Fournier, M. et Lécuyer, R. (2006). L'intelligence de l'enfant. Auxerre, France: Éditions Sciences Humaines.

Gardner, H. (1997). Les formes de l'intelligence. Paris, France: Éditions Odile Jacob.

Grégoire, J. (2000). L'évaluation clinique de l'intelligence de l'enfant. Théorie et pratique du WISC-III. Liège, Belgique: Mardaga.

Harter, S. (1982). The perceived competence scale for children. Child development, 53, 87-97. 
Hong, Y. Y., Chiu, C., Dweck, C. S., Lin, D. and Wan, W. (1999). Implicit theories, attributions, and coping: a meaning system approach. Journal of personality and social psychology, 77(3), 588-599.

Jöreskog, K. and Long, J. S. (1993). Introduction. In Kenneth A., Bollenet J. and Scott Long (eds): Testing Structural Equation Models. Newbury Park, California: SAGE publications.

Kamins, M. and Dweck, C. S. (1999). Person vs. process praise and criticism: Implications for contingent self-worth and coping. Developmental psychology, 35(3), 835-847.

Larivée, S. (2007). La nature du concept d'intelligence, un concept naturel. Dans S. Larivée (dir.), L'intelligence: tome 1, approches bio-cognitives, développementales et contemporaines (p. 2-3). Saint-Laurent, Québec: Édition du Renouveau pédagogique.

Larivée, S. et Morin, A. (2007). Qu'en pense le profane? Les théories implicites de l'intelligence. Dans S. Larivée (dir.), L'intelligence: tome 1, approches bio-cognitives, développementales et contemporaines (p. 3-6). Saint-Laurent, Québec: Édition du Renouveau pédagogique.

Marcoux, G. et Crahay, M. (2008). Mais pourquoi continuent-ils à faire redoubler? Essai de compréhension du jugement des enseignants. Revue des sciences de l'éducation, 30(3), 501-518.

Mickovska, A. (2009). A comparative analysis of Macedonian and English teachers' implicit theories of pupils' intelligence and motivation. Journal of european psychology students, 1(1), 1-10. Récupéré du site de la revue: http://w1.dorise.info/JCSE/paper_ detail.php ?pid=000820130110040923

Mugny, G. et Carugati, F. (1985). L'intelligence au pluriel. Les représentations sociales de l'intelligence et de son développement. Cousset (Fribourg), Suisse: Éditions Delval.

Piaget, J. et Inhelder, B. (1966). La psychologie de l'enfant. Paris, France: Presses universitaires de France.

Robins, R. W. and Pals, J. L. (2002). Implicit self-theories in the academic domain: implications for goal orientation, attributions, affect, and self-esteem change. Self and identity, 1, 313-336.

Rosenthal, R. et Jacobson, L. (1971). Pygmalion à l'école. Paris, France: Casterman.

Schunk, D. H. (1995). Implicit theories and achievement behavior. Psychological inquiry, 6, 311-314.

Smith, M. (1990). Teachers' beliefs about retention. In L. Shepard and M. Smith (eds.) Flunking grades: research and policies on retention (p.16-33). Bristol, United Kingdom: Falmer Press.

Sternberg, R. J. (2000). Handbook of intelligence. Cambridge, Massachusetts: Cambridge University Press.

Sternberg, R. J., Lautrey, J. and Lubbart, T. I. (2003). Models of intelligence. International perspectives. Washington, District of Columbia: American psychological association.

Vezeau, C., Bouffard, T. et Dubois, V. (2004). Relation entre la conception de l'intelligence et les buts d'apprentissage. Revue des sciences de l'éducation, 30(1), 9-25.

Vygotsky, L. S. (1978). Mind in Society. Cambridge, Massachusetts: Harvard University Press. 
152 Revue des sciences de l'éducation, volume 40, n 1, 2014

\section{Correspondance}

Elisabeth.Issaieva@unige.ch

Marcel.Crahay@unige.ch

\section{Contribution des auteurs}

Élisabeth Issaieva : $75 \%$

Marcel Crahay: 25\%

Ce texte a été révisé par: Charles Étienne Tremblay

Texte reçu le: 23 septembre 2012

Version finale reçue le: 14 avril 2014

Accepté le: 26 juin 2014 


\section{Annexe 1 - Tableau 5}

\section{Statistiques descriptives}

\begin{tabular}{|c|c|c|c|c|c|c|c|}
\hline \multicolumn{2}{|c|}{$\begin{array}{l}\text { Thématiques } \\
\text { et ses } \\
\text { dimensions }\end{array}$} & ltems & Moy & E. t. & Asymétrie & Aplatissement & Facteur \\
\hline \multirow{13}{*}{ 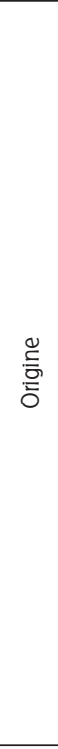 } & \multirow{3}{*}{ 芯 } & $\begin{array}{l}1 \text { Quand on est moins intelligent, il faut fournir plus d'efforts pour } \\
\text { réussir. }\end{array}$ & 3,08 & 0,75 & $-0,63$ & 0,39 & \\
\hline & & $\begin{array}{l}2 \text { La quantité d'efforts à fournir par un élève pour réussir n'est pas } \\
\text { liée à son intelligence. }\end{array}$ & 2,41 & 0,84 & 0,10 & $-0,56$ & \\
\hline & & $\begin{array}{l}3 \text { Le nombre d'heures que l'élève doit consacrer à travailler pour } \\
\text { réussir ne dépend pas de ses capacités intellectuelles. }\end{array}$ & 2,35 & 0,88 & 0,28 & $-0,59$ & \\
\hline & \multirow{10}{*}{$\frac{\cong}{\bar{y}}$} & 9 L'intelligence est fixée à la naissance. & 2,04 & 0,83 & 0,41 & $-0,44$ & 4 \\
\hline & & $\begin{array}{l}10 \text { Nous naissons tous approximativement avec les mêmes } \\
\text { capacités intellectuelles. C'est le milieu qui fait la différence. }\end{array}$ & 2,38 & 0,77 & 0,09 & $-0,35$ & 4 \\
\hline & & $\begin{array}{l}11 \text { À la naissance, hormis les cas de handicap sévère, tous les } \\
\text { enfants ont le même potentiel intellectuel. }\end{array}$ & 2,24 & 0,83 & 0,10 & $-0,65$ & 4 \\
\hline & & 12 Dès la naissance, certains sont plus intelligents que d'autres. & 2,60 & 0,88 & $-0,17$ & $-0,64$ & 4 \\
\hline & & $\begin{array}{l}13 \text { Dans le développement de l'intelligence, une grande part est } \\
\text { d'origine héréditaire. }\end{array}$ & 2,38 & 0,80 & 0,01 & $-0,48$ & \\
\hline & & $\begin{array}{l}14 \text { Chacun naît avec un certain potentiel d'intelligence qui se } \\
\text { développe via un phénomène de maturation biologique. }\end{array}$ & 2,52 & 0,75 & $-0,16$ & $-0,30$ & \\
\hline & & $\begin{array}{l}15 \text { Nous naissons chacun avec un certain potentiel intellectuel, à } \\
\text { l'image d'un réservoir délimité de capacités. }\end{array}$ & 2,27 & 0,78 & 0,19 & $-0,34$ & \\
\hline & & $\begin{array}{l}16 \text { À la naissance, nous disposons chacun d'un certain bagage } \\
\text { intellectuel qui va déterminer jusqu'où nous pouvons aller dans les } \\
\text { études. }\end{array}$ & 2,00 & 0,82 & 0,60 & $-0,05$ & \\
\hline & & 17 Chacun vient au monde avec un certain type d'intelligence. & 2,86 & 0,70 & $-0,17$ & $-0,15$ & \\
\hline & & $\begin{array}{l}18 \text { Dès la naissance, chaque individu a son propre style } \\
\text { d'intelligence. }\end{array}$ & 2,82 & 0,67 & $-0,30$ & 0,23 & \\
\hline \multirow{17}{*}{ 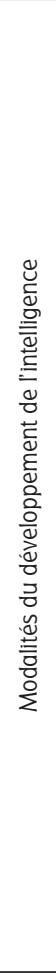 } & \multirow{6}{*}{ 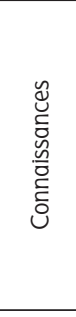 } & 19 Quand on lit beaucoup, on développe son intelligence. & 2,92 & 0,71 & $-0,29$ & $-0,05$ & \\
\hline & & 20 Plus on accumule des connaissances plus on devient intelligent. & 2,57 & 0,76 & 0,14 & $-0,39$ & 1 \\
\hline & & $\begin{array}{l}21 \text { En augmentant sa culture générale, on développe aussi son } \\
\text { intelligence. }\end{array}$ & 2,92 & 0,69 & $-0,43$ & 0,43 & 1 \\
\hline & & $\begin{array}{l}22 \text { Ce n'est pas parce qu'on lit beaucoup quon développe son } \\
\text { intelligence. }\end{array}$ & 2,52 & 0,63 & 0,05 & $-0,27$ & 1 \\
\hline & & $\begin{array}{l}23 \text { Ce n'est pas en accumulant de plus en plus de connaissances } \\
\text { qu'on développe son intelligence. }\end{array}$ & 2,52 & 0,66 & 0,17 & $-0,23$ & 1 \\
\hline & & $\begin{array}{l}24 \text { Ce n'est pas en améliorant sa culture générale qu'on développe } \\
\text { son intelligence. }\end{array}$ & 2,30 & 0,69 & 0,35 & 0,14 & 1 \\
\hline & \multirow{3}{*}{ 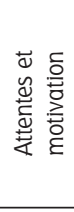 } & $\begin{array}{l}34 \text { L'enfant développera de plus en plus ses aptitudes si les adultes } \\
\text { croient en son intelligence. }\end{array}$ & 3,42 & 0,59 & $-0,44$ & $-0,69$ & \\
\hline & & $\begin{array}{l}35 \text { Plus l'enseignant exprime sa confiance dans les capacités de } \\
\text { l'enfant, plus celui-ci développera son intelligence. }\end{array}$ & 3,39 & 0,61 & $-0,45$ & $-0,65$ & \\
\hline & & $\begin{array}{l}36 \text { Impossible de développer son intelligence si on n'a pas } \\
\text { confiance en soi. }\end{array}$ & 3,11 & 0,73 & $-0,41$ & $-0,31$ & \\
\hline & \multirow{3}{*}{ 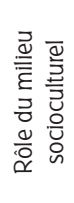 } & $\begin{array}{l}40 \text { Pour se développer, l'intelligence a besoin d'un climat affectif } \\
\text { favorable. }\end{array}$ & 3,33 & 0,69 & $-0,63$ & $-0,33$ & \\
\hline & & $\begin{array}{l}41 \text { L'intelligence se développe principalement grâce aux } \\
\text { stimulations du milieu. }\end{array}$ & 3,12 & 0,63 & $-0,34$ & 0,41 & 2 \\
\hline & & $\begin{array}{l}7 \text { Interagir avec des gens compétents aide à développer son } \\
\text { intelligence. }\end{array}$ & 3,40 & 0,58 & $-0,51$ & 0,26 & 2 \\
\hline & \multirow{5}{*}{ 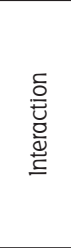 } & 42 L'intelligence se construit en interaction avec le milieu. & 3,24 & 0,57 & $-0,21$ & 0,46 & 2 \\
\hline & & $\begin{array}{l}4 \text { Ce n'est pas en interagissant avec les autres qu'on devient } \\
\text { intelligent. }\end{array}$ & 1,78 & 0,79 & 0,91 & 0,59 & \\
\hline & & 5 En discutant ses idées avec autrui, on développe son intelligence. & 3,43 & 0,65 & $-1,03$ & 1,47 & 2 \\
\hline & & $\begin{array}{l}6 \text { C'est en nous ouvrant et en nous intéressant au monde qui nous } \\
\text { entoure que nous devenons intelligents. }\end{array}$ & 3,30 & 0,67 & $-0,83$ & 1,18 & \\
\hline & & $\begin{array}{l}8 \text { C'est en faisant nos propres expériences que nous devenons } \\
\text { intelligents. }\end{array}$ & 3,23 & 0,66 & $-0,49$ & 0,20 & \\
\hline
\end{tabular}


154 Revue des sciences de l'éducation, volume 40, n 1, 2014

\begin{tabular}{|c|c|c|c|c|c|c|c|}
\hline \multicolumn{2}{|c|}{$\begin{array}{l}\text { Thématiques } \\
\text { et ses } \\
\text { dimensions }\end{array}$} & \multirow{2}{*}{\begin{tabular}{|l|} 
Items \\
43 Ce n'est pas parce qu'on est intelligent qu'on comprend plus vite \\
que les autres.
\end{tabular}} & \multirow{2}{*}{\begin{tabular}{|l|} 
Moy \\
2,34
\end{tabular}} & \multirow{2}{*}{\begin{tabular}{l|} 
E. t. \\
0,74
\end{tabular}} & \multirow{2}{*}{$\begin{array}{c}\text { Asymétrie } \\
-0,02\end{array}$} & \multirow{2}{*}{\begin{tabular}{c|} 
Aplatissement \\
$-0,38$
\end{tabular}} & \multirow[t]{2}{*}{ Facteur } \\
\hline \multirow{12}{*}{$\begin{array}{l}\frac{0}{5} \\
\stackrel{0}{Z}\end{array}$} & \multirow{6}{*}{ 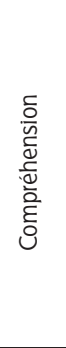 } & & & & & & \\
\hline & & $\begin{array}{l}44 \text { Ce n'est pas parce qu'on est moins intelligent qu'on est plus lent } \\
\text { à comprendre }\end{array}$ & 2,48 & 0,70 & $-0,24$ & $-0,24$ & \\
\hline & & $\begin{array}{l}45 \text { Être plus lent à comprendre n'est pas un manque d'intelligence, } \\
\text { chacun a son propre rythme. }\end{array}$ & 3,01 & 0,66 & $-0,12$ & $-0,31$ & \\
\hline & & $\begin{array}{l}37 \text { Les enfants intelligents nont pas besoin de recevoir de longues } \\
\text { explications avant de comprendre. }\end{array}$ & 2,97 & 0,75 & $-0,24$ & $-0,51$ & 5 \\
\hline & & 38 Un élève intelligent est un élève qui comprend rapidement. & 2,95 & 0,76 & $-0,12$ & $-0,76$ & 5 \\
\hline & & $\begin{array}{l}39 \text { Quand on est moins intelligent, il faut plus de temps pour } \\
\text { comprendre. }\end{array}$ & 2,99 & 0,68 & $-0,18$ & $-0,25$ & 5 \\
\hline & \multirow{6}{*}{ हैँ } & $\begin{array}{l}28 \text { Bonne mémoire et intelligence ne vont pas nécessairement de } \\
\text { pair. }\end{array}$ & 2,94 & 0,80 & $-0,42$ & $-0,21$ & \\
\hline & & $\begin{array}{l}29 \text { Le fait d'avoir une bonne ou une mauvaise mémoire n'est pas } \\
\text { du tout lié à l'intelligence. }\end{array}$ & 2,99 & 0,76 & $-0,26$ & $-0,52$ & \\
\hline & & $\begin{array}{l}30 \text { Être capable de mémoriser une grande quantité d'informations } \\
\text { n'est pas une preuve d'intelligence. }\end{array}$ & 3,16 & 0,70 & $-0,41$ & $-0,23$ & \\
\hline & & 31 Une bonne mémoire est généralement un signe d'intelligence. & 2,17 & 0,72 & 0,24 & $-0,07$ & \\
\hline & & 32 Les élèves intelligents ont généralement une bonne mémoire. & 2,86 & 0,57 & $-0,52$ & 1,30 & \\
\hline & & $\begin{array}{l}33 \text { Les individus intelligents sont capables de mémoriser une } \\
\text { grande quantité d'informations. }\end{array}$ & 2,78 & 0,61 & $-0,25$ & 0,26 & \\
\hline \multirow{3}{*}{ 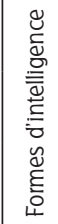 } & & $\begin{array}{l}25 \text { Les élèves doués en mathématiques et en sciences sont } \\
\text { souvent moins doués pour les relations sociales. }\end{array}$ & 1,83 & 0,73 & 0,43 & $-0,48$ & 3 \\
\hline & & $\begin{array}{l}26 \text { Les élèves doués pour les relations sociales sont souvent moins } \\
\text { doués en mathématiques et en sciences. }\end{array}$ & 1,79 & 0,71 & 0,41 & $-0,61$ & 3 \\
\hline & & $\begin{array}{l}27 \text { Les élèves qui ont une intelligence plutôt littéraire éprouvent des } \\
\text { difficultés dans les domaines scientifiques. }\end{array}$ & 2,17 & 0,76 & $-0,02$ & $-0,70$ & 3 \\
\hline
\end{tabular}


Annexe 2

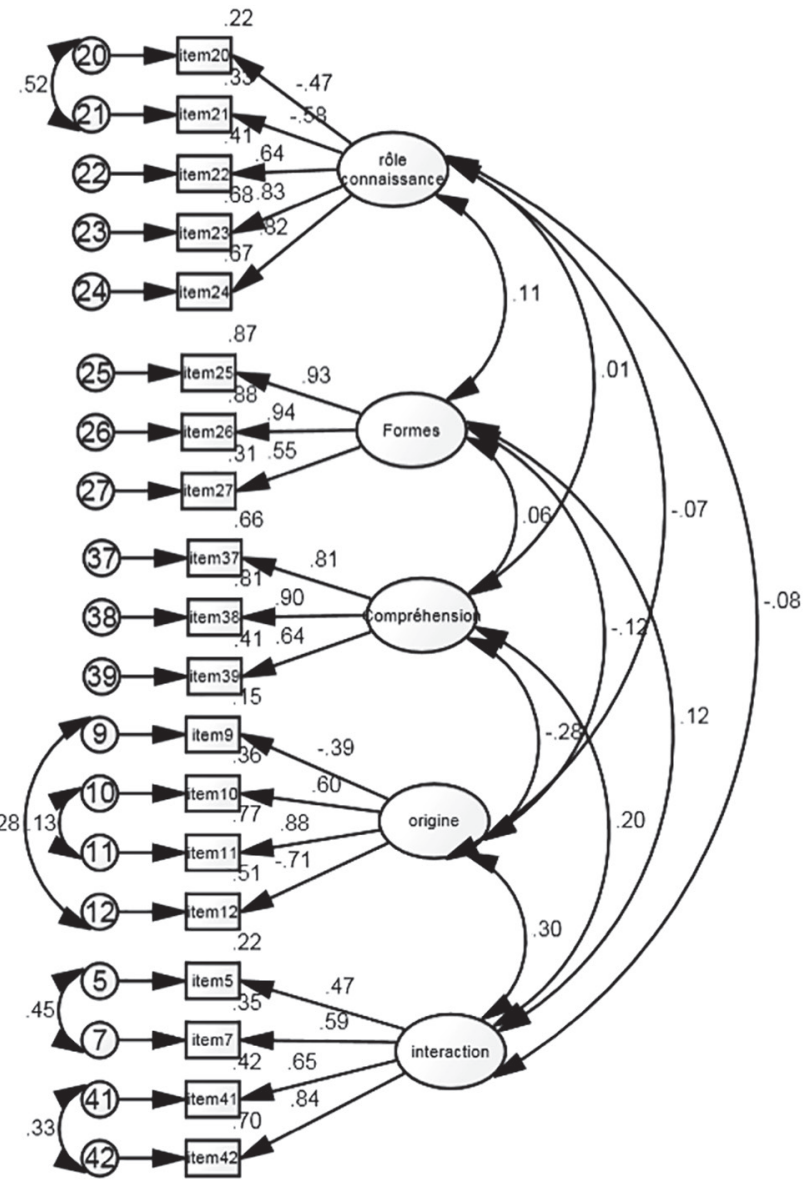

Figure 1. Analyse factorielle confirmatoire 
156 Revue des sciences de l'éducation, volume 40, n 1, 2014

Annexe 3

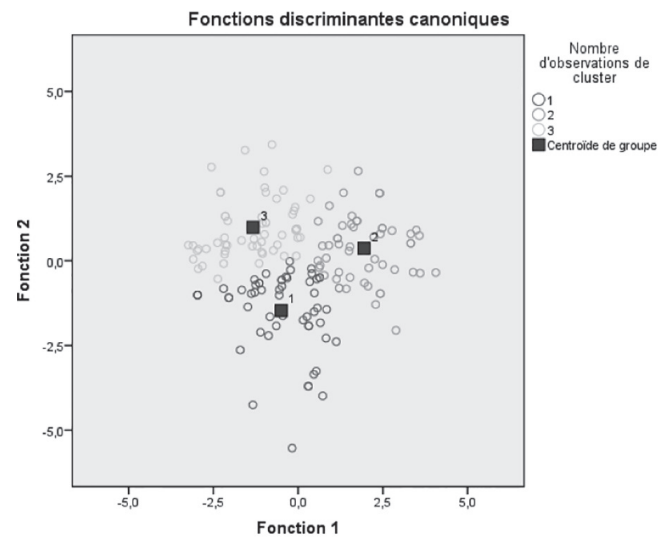

Figure 2. Représentation graphique des groupes

Annexe 4

\section{Récapitulatif du modèle}

\begin{tabular}{|l|l|}
\hline Algorithme & TwoStep \\
\hline Entrées & 5 \\
\hline Clusters & 3 \\
\hline
\end{tabular}

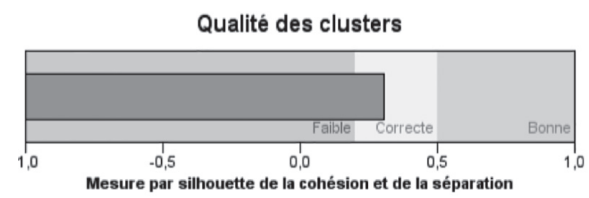

Figure 3. Qualité des groupes 